\title{
Modelagem de redes logísticas com custos de inventário calculados a partir da cobertura de estoque
}

\author{
Ricardo Hamad ${ }^{a *}$, Nicolau Dionísio Fares Gualda ${ }^{b}$ \\ a*ricardo.hamad@usp.br, USP, Brasil \\ bngualda@usp.br, USP, Brasil
}

\begin{abstract}
Resumo
A consideração dos custos de inventário tem se tornado cada vez mais importante na análise dos trade-offs e na tomada de decisões sobre redes logísticas. 0 artigo propõe uma metodologia para tratamento do custo de inventário a partir da cobertura de estoque esperada em cada instalação, incorporada na modelagem matemática da localização de fábricas e/ou centros de distribuição em redes logísticas com vários elos, representando uma nova versão do modelo apresentado em Hamad e Gualda (2008). As principais contribuições desta metodologia em relação a outros métodos encontrados na literatura são a facilidade de aplicação do método, a inclusão de restrições ligadas à capacidade de armazenagem e a consideração do custo total de inventário, e não apenas do custo relacionado aos produtos modelados.
\end{abstract}

Palavras-chave

Otimização de redes logísticas. Configuração de redes logísticas. Giro de inventário. Custos de inventário.

\section{Introdução}

Conforme atesta Standage (2009), a manutenção de reservas na forma de estoque se mostrou um dos principais fatores para a sobrevivência e evolução do ser humano. Seja na forma de colheitas e restos de animais armazenados em cavernas, ou mesmo na forma de gordura presente no corpo humano, os estoques permitiram que o homem se tornasse menos dependente de estar próximo às fontes de comida, o que levou a que essa espécie pudesse descer das árvores e se organizar em torno de grupos fixos em locais mais propícios para o seu desenvolvimento. Sem os estoques, o ser humano seria obrigado a se deslocar constantemente em busca de novas fontes de alimento, à medida que estas fossem se esgotando. Desde o início da sua história, a humanidade tem usado estoques de variados recursos, de modo a suportar o seu desenvolvimento e sobrevivência, tais como ferramentas e alimentos (GARCIA et al., 2006).

Atualmente, os estoques representam mais de 50\%, em média, do ativo circulante das empresas
(CHRISTOPHER, 2002) e constitui um dos principais fatores de análise nas decisões sobre redes logísticas. Dentre os custos de uma rede logística, o fator inventário, ou seja, a relação de bens disponíveis em estoque, é um dos mais importantes por afetar diretamente os dois indicadores principais do desempenho de uma cadeia de suprimentos: o nível de serviço ao cliente na sua perspectiva de disponibilidade do produto e o giro de estoque da cadeia. Enquanto o primeiro mede o bom gerenciamento da rede logística em relação ao seu principal foco externo, os consumidores, o segundo indicador representa o quão bem está sendo administrado um dos ativos internos mais importantes das companhias, já que o estoque tem se tornado o mais representativo em termos de capital empregado e responsável pelos maiores desperdícios. Como exemplo disso pode-se citar uma pesquisa da AMR Research (Boston), publicada em julho/2000 e comentada por Crandall e Crandall 
(2003), que projetava um excesso de inventário de bens de consumo de US\$ 60 bilhões nos EUA e US\$ 120 bilhões no mundo para o final de 2000, o que representaria cerca de $6 \%$ do total do inventário disponível projetado para aquele período.

Os modelos de localização de fábricas e centros de distribuição (CDs) tentam otimizar os custos ligados à movimentação dos materiais entre os elos e, para que todos os custos importantes sejam considerados, a inclusão dos custos dos estoques não pode ser esquecida. Um bom gerenciamento de estoques reduz o inventário em excesso, melhorando, dessa forma, o fluxo de caixa das empresas. Talvez por conta dessa percepção crescente da importância dos estoques e, consequentemente, do resultante impacto no fluxo de caixa, modelos mais recentes de localização passaram a incluir os custos de inventário na função objetivo: Bhutta (2001), Smits (2001), Nozick e Turnquist (2001), Flipo (2000), Flipo e Finke (2001), Syam (2002), Goetschalckx, Vidal e Dogan (2002), Bhutta et al. (2003), Vallim Filho (2004) e Brito Junior (2004). Mas nota-se em todos esses modelos uma consideração apenas parcial dos custos totais de inventário.

0 objetivo deste trabalho é propor uma metodologia relativamente simples para tratamento dos custos totais de inventário de uma rede logística em um modelo de localização a partir do giro de estoque em cada ponto da cadeia representando um aprimoramento do modelo apresentado em Hamad e Gualda (2008). 0 modelo de Programação Linear Inteira Mista (PLIM) baseado em uma planilha eletrônica do Microsoft Excel é apresentado, assim como os resultados de sua aplicação em uma empresa multinacional do segmento químico.

\section{Revisão da literatura}

Modelos de localização têm sido estudados já há algum tempo, e os primeiros trabalhos encontrados na literatura remetem à década de 60: Kuehn e Hamburger (1963), Efroymson e Ray (1966) e Spielberg (1969). Modelos de inventário também têm sido estudados há muito tempo, mas a associação dos modelos de inventário aos modelos de localização é bastante recente. Harris (1913), por exemplo, determinou a fórmula para o cálculo da quantidade ótima de pedido a partir do custo de inventário e do custo de se colocar um pedido de compras/produção para demandas conhecidas e que apresentam certa regularidade. Essa fórmula foi explorada por Wilson (1934) e tem sido largamente usada para cálculo de lote ideal ou, no inglês, EOQ (Economical Order Quantity). Entretanto, apenas recentemente autores têm usado o tamanho do lote transportado para os depósitos (calculado pela taxa de produção multiplicada pela frequência de entrega) para determinar o custo de inventário em modelos de localização (DOGAN; GOESTSCHALCKX, 1999).

Smits (2001) faz uma vasta revisão dos modelos de localização existentes e apresenta uma taxonomia que inclui as diversas formas de tratamento dos custos de inventário. Segundo o autor, modelos de localização que tratam de custos de inventário são recentes, mas vêm ganhando importância. Em outras revisões de modelos de localização, tais como Geoffrion e Powers (1995), Owen e Daskin (1998), Goetschalckx, Vidal e Dogan (2002), Bhutta (2004) e Revelle e Eiselt (2005), o tratamento dos estoques nos diversos modelos disponíveis não é sequer avaliado.

Arrow, Karlin e Scarf (1958) apresentam as várias motivações para se ter inventário (motivos transacionais, por precaução e por especulação) e alguns modelos de inventário. Nahmias (1993) apresenta as várias formas de tratar os custos de inventário para modelos de localização para casos de um elo na cadeia e um único produto. Eppen (1979) deriva uma relação entre a quantidade de centros de distribuição (CDs) paralelos e a quantidade de estoque de segurança para os modelos com apenas um elo na cadeia e demandas idênticas em cada CD: o novo estoque do sistema é igual à multiplicação do estoque de segurança para um CD pela raiz quadrada do número total de CDs. Nozick e Turnquist (2001) usam essa fórmula para o cálculo do estoque de segurança no modelo de localização de CDs, considerando que para $n \geq 15$ a função raiz quadrada converge para uma função linear.

Smits (2001) propõe modelos de localização que tratam do custo de inventário partindo de considerações como: ordens dos clientes obedecem a um processo de reorder point, a demanda é dada por uma função de distribuição arbitrária e o lead time inclui também o tempo de espera devido à falta de estoque no armazém central. 0 nível médio de inventário é calculado a partir da distribuição da chegada de ordens, do lead time, da taxa de entregas e do tamanho dos lotes de entrega, mas só inclui os estoques dos produtos que estão sendo transportados. Essa limitação acontece também no modelo apresentado por Syam (2002), onde o inventário é calculado a partir do cycle time e do fluxo em determinado arco para os vários períodos e produtos considerados. No modelo de Hadjinicola e Kumar (2002) o inventário médio é aproximado pela metade da demanda do período, e no modelo apresentado por Gross, Pinkus e Soland 
(1981) o nível médio de inventário e a quantidade de stockout são calculados a partir do modelo de Clark (1958), usando uma função de distribuição de probabilidade geral para o tamanho da ordem por unidade de tempo.

Modelos dinâmicos, como os de Mohamed (1999), Dogan e Goettschalckx (1999) e Arntzen et al (1995), tendem a usar o inventário do final dos períodos para o cálculo do custo de inventário. Se o período t é grande (um mês ou maior), isso pode levar a grandes erros, já que o inventário do final do período tende a ser bem menor que o inventário médio do período. Além disso, os inventários de produtos intermediários e matérias-primas não foram considerados nos modelos analisados.

Apenas em poucos modelos, como, por exemplo, em Arntzen et al. (1995), Dogan e Goetschalckx (1999), Smits (2001) e Brito Junior (2004), os inventários em trânsito são considerados, mas somente os inventários dos produtos que estão sendo transferidos. Produtos intermediários e matérias-primas em trânsito não são levados em conta.

Shapiro (2001) ensina como incorporar o gerenciamento do inventário em modelos estratégicos para a configuração de redes logísticas e afirma que a consideração usual de inventário zero no final do período é uma aproximação não correta, se considerarmos que, na grande maioria dos casos, existirá produção ou distribuição nos períodos seguintes ao considerado.

Ballou (1998) comenta que o procedimento agregado mais comum para controle do estoque é o giro de estoque dado pelo quociente de vendas anuais sobre o investimento médio em estoque para o mesmo período de tempo. 0 autor afirma que a popularidade da medida vem de sua simplicidade e da disponibilidade pronta dos dados, já que os estoques são, por imposição legal e pela necessidade de se ter um bom gerenciamento do negócio, controlados de forma detalhada pelas empresas. A cobertura em dias é dada pelo inverso do giro de estoque multiplicado por 365 . 0 conceito clássico usado para medição da cobertura exige, portanto, a divisão do inventário médio total anual pelo total do custo da mercadoria vendida. A cobertura é normalmente chamada de DOH (Days-On-Hand) em empresas multinacionais, pois esse termo é o mais utilizado em língua inglesa para designar a cobertura dos estoques (FINANCIAL EDUCATION, 2007).

A partir dos modelos encontrados na literatura e abordados neste estudo, os seguintes problemas nos tratamentos de inventário podem ser apontados:
- 0 inventário total em cada elo da cadeia (que inclui, além de produtos acabados, matérias-primas, produtos intermediários, itens de manutenção, insumos de manufatura, estoques de segurança em geral e inventários em trânsito) não é contemplado ou é contemplado apenas parcialmente;

- As restrições de capacidade de armazenagem são normalmente consideradas apenas indiretamente, através das restrições de capacidade de manuseio dos produtos nos CDs ou nas fábricas intermediárias; e

- Os modelos que tentam incluir parcelas representativas dos inventários tornam o modelo bastante complexo, restringindo ou impedindo o uso de modelos de programação linear.

\section{Proposta para tratamento dos custos de inventário em modelos de localização com vários elos}

A principal contribuição no modelo ora proposto é a forma de cálculo dos custos relacionados ao inventário dentro de um modelo de localização com vários elos na cadeia. A aplicação de uma metodologia próxima da que se está propondo neste estudo foi feita pela primeira vez em Hamad e Gualda (2006). 0 objetivo maior do modelo apresentado naquele trabalho foi a otimização de custos em cadeias logísticas de vários elos levando em conta os impostos gerados pela rede logística da empresa em estudo. A modelagem dos inventários no pico e as restrições sobre capacidade de armazenagem propostas neste trabalho não foram incorporadas ao modelo naquela oportunidade. Na verdade, nenhum outro modelo encontrado na literatura considera o giro e/ou cobertura do estoque no tratamento dos custos do inventário.

Os inventários médios de uma fábrica/CD são estimados a partir dos valores esperados de cobertura de estoque em dias. A cobertura em dias será chamada, neste artigo, de DOH (Days-On-Hand) e o custo da mercadoria vendida será denominado Cost of Goods Sold ou COGS. 0 DOH será calculado pelo quociente do inventário médio anual sobre o COGS transferido para o elo seguinte da cadeia no período de um ano, ou seja:

$D O H=\frac{\overline{\text { Inventário_anual }}}{\sum \text { COGS }} \times 365$

Como, por premissa recorrente nos modelos matemáticos de localização, o volume que sai de cada elo em unidade padrão deverá ser o mesmo volume que ingressa nas fábricas ou CDs, o inventário médio em determinado elo $f$ da cadeia pode ser dado por: 
$\overline{\text { Inventário_anual }}_{f}=\frac{\mathrm{DOH}_{f} \times \sum \mathrm{COGS}}{365}, \forall f$

onde:

$\mathrm{DOH}_{f}=$ cobertura esperada para o elo $f$ da cadeia

$\overline{\text { Inventário_anual }}_{f}=$ Inventário médio do elo $f$ no ano $\sum \mathrm{COGS}_{f}=\mathrm{COGS}$ que ingressa no elo $f$ mais a agregação de valor nesse elo.

0 custo a ser incluído na função objetivo compreende o custo de carregar o inventário $(\mathrm{Cl})$ em cada elo $f$, que é dado pela seguinte fórmula:

$C I=\overline{\text { Inventário_anual }}_{f} \times C C$

onde:

$\mathrm{CC}=\mathrm{o}$ custo em porcentual dos estoques para carregar o inventário calculado a partir do WACC (Weighted Average Cost of Capital) somado ao custo de armazenamento

A alternativa para tratamento de inventário sugerida neste estudo leva em conta apenas o giro de estoque esperado em cada elo da cadeia, o que simplifica a modelagem e permite que seja mantida a linearidade da função objetivo e restrições. Dessa forma, no caso de vários elos na cadeia logística, há que se preocupar apenas com a definição da cobertura esperada (em dias) em cada elo, o que pode ser calculado a partir de dados reais, para elos já existentes, aplicando-se a fórmula (2), ou estimado a partir de parâmetros. Algumas considerações sobre como estimar o DOH a partir de parâmetros são apresentadas no item 4 deste artigo.

Como exemplo de aplicação desta metodologia, considerou-se o caso de uma empresa química que possui fábricas de produto intermediário (princípio ativo), fábricas de produto acabado e centros de distribuição (CDs) usados para a entrega de seus produtos acabados. 0 resultado do modelo determina quais fábricas de produto intermediário (ingrediente ativo), fábricas de produto acabado e quais CDs deverão ser selecionados pela empresa, assim como os volumes a serem transferidos entre cada elo, de forma a minimizar o total de seus custos fixos e variáveis mais importantes. Para o tratamento dos custos de carregamento do inventário adotou-se a metodologia proposta neste artigo.

A representação da cadeia de suprimentos contemplada encontra-se na Figura 1.

Para esse caso, foi proposto um modelo matemático estático de localização de instalações com quatro elos na cadeia, a saber:

- Primeiro elo: representa as fábricas de produto intermediário (com índices $i$ ) que produzem o

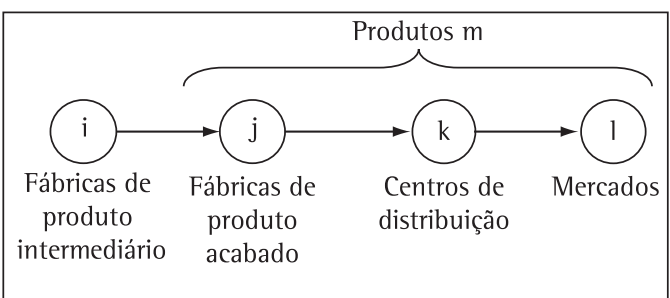

Figura 1. Cadeia de suprimento com quatro elos.

ingrediente ativo ou matéria-prima básica dos produtos acabados;

- Segundo elo: as fábricas de produto acabado $j$ que produzem o produto $m$;

- Terceiro elo: os centros de distribuição - CDs (índice $k$ ); e

- Quarto elo: os mercados $l$ que consomem os produtos finais $m$.

Para caracterização dos custos da empresa e para a definição das restrições aplicáveis entre os vários elos, é mandatório considerar-se os valores de demanda, capacidades em cada elo, custos variáveis relacionados à movimentação e à armazenagem e os custos fixos incorridos em cada ponto da cadeia. Com isso, os seguintes parâmetros foram adotados:

- Dem $_{m l}=$ demanda do produto $m$ no mercado l;

- Cap_arm $=$ capacidade de armazenagem da fábrica de produto acabado $j$;

- Cap_arm ${ }_{k}=$ capacidade de armazenagem do Centro de Distribuiç̧ão $k$;

- Cap_pro ${ }_{i}=$ capacidade de produção da fábrica intermediária $i$;

- Cap_pro ${ }_{j}=$ capacidade de produção da fábrica de produto acabado $j$;

- Cap_man ${ }_{k}=$ capacidade de manuseio do centro de distribuição $k$;

- CusT $_{i j}=$ custo unitário em \$/unidade padrão para transportar o produto intermediário $i$ para o centro produtor $j$;

- CusT $_{j k}=$ custo unitário médio em \$/unidade padrão para transportar o produto do centro produtor $j$ para o CD $k$ (considera-se que haverá consolidação de carga com diferentes produtos $m$ e, portanto, é considerado apenas o custo médio de transferência);

- CusD $_{m k l}=$ custo unitário médio em \$/unidade padrão para distribuir o produto final $m$ do CD $k$ para o mercado $l$;

- CusB $_{i}=$ custo unitário (\$/unidade padrão) do produto intermediário fabricado na fábrica $i$; 
- CusB $_{j m}=$ custo unitário (\$/unidade padrão) a ser agregado na fábrica $j$ para transformar o produto intermediário em produto acabado $\mathrm{m}$;

- CusB $_{m k}=$ custo unitário (\$/unidade padrão) a ser agregado no CD $k$ para manusear o produto acabado $m$;

- CusFix $_{j}=$ custo fixo (\$/ano) da fábrica $j$ (o custo fixo, indiretamente, representa também o investimento necessário, pois a depreciação respectiva deverá fazer parte desse valor; caso se opte por subcontratação, o que evitaria os investimentos necessários para a construção de uma fábrica, o custo fixo deverá ser apenas aquele estipulado no contrato de prestação de serviços de industrialização);

- CusFix $_{k}=$ custo fixo (\$/ano) do CD $k$ (caso seja considerada apenas a contratação de terceiros, o que evitaria os investimentos necessários para a construção de um $\mathrm{CD}$, o custo fixo deverá ser apenas aquele estipulado no contrato de prestação de serviços de armazenagem e manuseio dos itens);

- CusM $_{j}=$ custo médio do produto intermediário na fábrica $j$;

- $\mathrm{DOH}_{j}=$ Days-On-Hand (cobertura de estoque) da fábrica $j$ em dias;

- $\mathrm{DOH}_{k}=$ Days-On-Hand (cobertura de estoque) do CD $k$ em dias;

- $\mathrm{P}_{\mathrm{f}}=\%$ adicional do inventário de pico que chega à fábrica $j \mathrm{em}$ relação ao inventário médio;

- $\mathrm{P}_{k}=\%$ adicional do inventário de pico que chega ao depósito $k$ em relação ao inventário médio; e

- $\mathrm{CC}=$ custo de carregar o estoque em \% do valor do estoque, que é dado por:

$C C=C F+C M$

onde:

CF: custo de oportunidade financeira (em \% do valor do estoque);

CM: custo envolvido com o armazenamento do material (em \% do valor do estoque).

0 custo de oportunidade financeira (ou custo de capital) é considerado como sendo a taxa de desconto para os fluxos de caixa dos ativos, descontado o imposto de renda. A forma de cálculo do custo de capital normalmente utilizada pelas empresas baseia-se na metodologia WACC (Weighted Average Cost of Capital), que leva em conta os custos do patrimônio líquido e os custos do passivo da empresa descontados do imposto de renda. 0 custo de armazenamento combina uma série de outros custos associados à manutenção física dos estoques (BALLOU, 1998).
As variáveis do modelo que representam o fluxo de materiais entre os vários elos são:

- Trns $_{i j}=$ volume do princípio ativo a ser transferido da fábrica $i$ para a fábrica $j$;

- Trns $_{j k m}=$ volume do produto acabado $m$ a ser transferido da fábrica $j$ para o CD $k$;

- Dstr $_{m k l}=$ volume do produto $m$ a ser distribuído do CD $k$ para o mercado $l$.

Além disso, as seguintes variáveis binárias também precisam ser consideradas para que o resultado do modelo possa sugerir quais instalações devem ser usadas e quais devem ser preteridas:

- $\mathrm{Z}_{j}$ = assume valor 1 se a fábrica $j$ for aberta, ou 0 em caso contrário;

- $\mathrm{Z}_{f}=$ assume valor 1 se a fábrica $f$ for aberta, ou 0 em caso contrário; e

- $\mathrm{Z}_{k}=$ assume valor 1 se o CD $k$ for aberto, ou 0 em caso contrário.

0 estoque médio na fábrica $j(\mathrm{IMj})$ é dado por:

$$
\begin{aligned}
& \mathrm{IM}_{j}=\frac{\mathrm{DOH}_{j}}{365} \times\left(\sum_{i}\left(\left(\text { Cus }_{i j}+\text { CusB }_{i}\right) \times \operatorname{Trns}_{i j}\right)\right. \\
& \left.+\left(\sum_{m} \sum_{k}\left(\text { CusB }_{j m}\right) \times \operatorname{Trns}_{j k m}\right)\right), \forall j
\end{aligned}
$$

0 estoque médio em $k\left(\mathrm{IM}_{k}\right)$ é dado por:

$$
\begin{gathered}
\mathrm{IM}_{k}=\frac{\mathrm{DOH}_{k}}{365} \times \sum_{j} \sum_{m}\left(\left(\operatorname{Cus}_{j k}+\mathrm{CusB}_{k m}+\right.\right. \\
\left.\left.\mathrm{CusB}_{j m}+\mathrm{CusM}_{j}\right) \times \operatorname{Trns}_{j k m}\right), \forall k
\end{gathered}
$$

onde, para manter-se a linearidade da função, foi feita uma aproximação como segue:

$$
\mathrm{CusM}_{j}=\frac{\sum_{i} \sum_{j}\left(\left(\mathrm{Cusb}_{i}+\mathrm{Cust}_{i j}\right) \times \operatorname{Trns}_{i j}\right)}{\sum_{m} \sum_{l} \text { Dem }_{m l}} \text {, igual para } \forall j
$$

Importante notar que, no caso do cálculo do estoque para o depósito $k$, foi necessário aproximar o valor do COGS unitário do produto intermediário a partir do seu custo médio (custo médio do princípio ativo/matéria-prima produzida em $i+$ os custos de transporte até $j$ ). lsso é possivel considerando-se a hipótese de que a diferença de custo entre as fábricas $i$ não é muito diferente e o custo de transporte não é muito significativo, o que é uma realidade no caso de produtos de alto valor agregado, como o de empresas químicas. Caso os custos sejam muito diversos, essa premissa precisa ser revista e o modelo devidamente modificado.

A função objetivo deve considerar todos os custos principais da cadeia de suprimentos: custos de movimentação dos materiais, custos fixos nos vários elos e os custos para se carregar os estoques: 
$\min C T$

$C T=\sum_{i} \sum_{j}\left(\left(C u s T_{i j}+C u s B_{i}\right) \times \operatorname{Trns}_{i j}\right) \longrightarrow(8.1)$

$+\sum_{j} \sum_{k} \sum_{m}\left(\left(\operatorname{Cus}_{j k}+\mathrm{CusB}_{j m}\right) \times \operatorname{Trns}_{j k m}\right) \longrightarrow(8.2)$

$+\sum_{m} \sum_{k} \sum_{l}\left\{\left[\right.\right.$ Cust $_{m k l}+$ CusB $\left._{m k}\right] \times$ Dstr $\left._{m k l}\right\} \longrightarrow(8.3)$

$+\sum_{j}\left(\right.$ CusFix $\left._{j} \times Z_{j}\right)+\sum_{k}\left(\right.$ CusFix $\left._{k} \times Z_{k}\right) \longrightarrow(8.4)$

$+\left(I M_{j}+I M_{k}\right) \times C C \longrightarrow(8.5)$

onde:

(8.1) - custo de transferência das fábricas $i$ para as fábricas $j$;

(8.2) - custo de transferência dos produtos $m$ das fábricas $j$ para os CDs $k$;

(8.3) - custo de distribuição dos produtos $m$ a partir dos CDs $k$ para os mercados $l$;

(8.4) - total dos custos fixos da(s) fábrica(s) ou depósito(s) indicados para serem abertos; e

(8.5) - custo para carregar os estoques (carrying cost) nas fábricas $j$ e nos CDs $k$.

Limitações ligadas à capacidade dos vários elos e a consideração de que o balanço de materiais em cada elo é zero, ou seja, o estoque médio mantido em cada ponto da cadeia para cobrir os desvios de suprimento ou demanda e para a manutenção das fábricas ou CDs não pode ser aumentado, geram as seguintes restrições:

Atender a demanda prevista:

$$
\operatorname{Dem}_{m l} \leq \sum_{k} \text { Dstr }_{m k l} \quad \forall l, m .
$$

Capacidade produtiva do princípio ativo/matériaprima na fábrica $i$ :

$$
\sum_{j} \operatorname{Trns}_{i j} \leq \mathrm{Cap}-\text { pro }_{i} \quad \forall i .
$$

Capacidade produtiva dos produtos acabados $m$ na fábrica $j$ :

$$
\sum_{k} \sum_{m} \operatorname{Trns}_{j k m} \leq \mathrm{Cap}-\text { pro }_{j} \times Z_{j} \quad \forall j .
$$

Capacidade de manuseio no depósito $k$ :

$$
\sum_{l} \sum_{m} D_{s t r_{m k l}} \leq C a p_{-} \operatorname{man}_{k} \times Z_{k} \quad \forall k .
$$

Capacidade de armazenagem na fábrica $j$ :

$$
I M_{j} \times\left(1+P_{j}\right) \leq C a p \_a r m_{j} \quad \forall j .
$$

Capacidade de armazenagem no CD k:

$$
I M_{k} \times\left(1+P_{k}\right) \leq \text { Cap_arm }{ }_{k} \quad \forall k .
$$

Balanço do princípio ativo na fábrica $j$ :

$$
\sum_{i} \text { Trns }_{i j}=\sum_{k} \sum_{m} \operatorname{Trns}_{j k m} \quad \forall j .
$$

Balanço do produto acabado no depósito $k$ :

$$
\sum_{j} \sum_{m} \operatorname{Trns}_{j k m}=\sum_{l} \sum_{m} \text { Dstr }_{m k l} \quad \forall k .
$$

Não negatividade:

$$
\begin{aligned}
& \operatorname{Trns}_{i j} \geq 0 \quad \forall i, j . ; \quad \operatorname{Trns}_{j k m} \geq 0 \quad \forall j, k, m . ; \\
& D_{s t r_{m k l}} \geq 0 \quad \forall m, k, l .
\end{aligned}
$$

Variáveis binárias:

$Z_{j}=\left\{\begin{array}{l}1, \text { se a fábrica } j \text { for aberta } \\ 0, \text { em caso contrário }\end{array} \quad \forall j\right.$

$Z_{k}=\left\{\begin{array}{l}1, \text { se o depósito } k \text { for aberto } \\ 0, \text { em caso contrário }\end{array} \forall k\right.$

\section{Considerações sobre a cobertura de estoque (DOH)}

A cobertura de estoque (DOH) pode ser calculada a partir da fórmula (1) quando uma determinada fábrica de produto acabado ou um CD já existe. Caso sejam analisadas novas alternativas de fábricas ou CDs, a cobertura poderá ser estimada a partir da consideração dos seguintes fatores:

- Volumes de matéria-prima/princípio ativo ou produtos acabados em trânsito esperados: quanto maior os tempos de trânsito envolvidos na chegada das matérias-primas/princípios ativos ou produtos acabados, maior será o inventário em trânsito e, consequentemente, isso será refletido em uma cobertura com maior número de dias;

- Flexibilidade da unidade produtiva ou CD: a boa administração do estoque e, consequentemente, a melhoria no giro de estoque depende em grande parte da qualificação dos funcionários, da disponibilidade de recursos (pessoas e ferramentas) e da implantação de boas práticas de planejamento da produção e da demanda;

- Sazonalidade da demanda do elo seguinte da cadeia: caso a distribuição seja contínua, o giro de estoque tende a ser maior, o que implica menores dias de cobertura. Caso haja sazonalidade, o inventário chamado de build-up fará com que a cobertura tenha que ser, em média, maior;

- Variabilidade da demanda e do fornecimento: quanto maior a confiabilidade dos fornecedores e a precisão das previsões de vendas, menor será a necessidade de se manter estoques de segurança, o que implica menor cobertura de estoque; 
- Estoques de MRO (Maintenance, Repair and Operations): quanto maior a necessidade de peças de reposição, materiais de segurança e insumos, maior será o total de inventário o que, consequentemente, leva a uma maior cobertura de estoques.

Dependendo do segmento de negócio, da maturidade dos produtos e do mercado, podem-se adotar valores de DOH a partir de benchmark com empresas similares. A partir dos balanços dessa empresas ou de anuários de entidades de classe como Abiquim, Sindimaq etc., o giro de estoque e, consequentemente, a cobertura esperada podem ser facilmente calculados.

Informações desse tipo podem também ser obtidas em sites como www.bizstats.com, por exemplo, que fornece índices financeiros e dados estatísticos sobre negócios nos EUA.

\section{Aplicação}

0 modelo proposto usa programação linear inteira mista (PLIM) e a entrada de dados é feita em uma planilha eletrônica do aplicativo Microsoft Excel, para facilidade de utilização. Essa nova versão do modelo foi testada, como no caso da versão anterior, em uma empresa química transnacional do segmento agrícola.

A rede logística modelada considerou quatro famílias de produtos acabados, três fábricas intermediárias que produziam o ingrediente ativo, três fábricas de produto acabado, dez centros de distribuição e 27 mercados. Dessa forma, o modelo ficou com 1.240 variáveis, 214 restrições e 22 variáveis binárias. Para essa quantidade de variáveis e restrições, o aplicativo suplementar Solver do MSExcelse mostrou insuficiente para a resolução do problema, o que tornou necessária a aquisição do suplemento Premium Solver Platform for Excel da Frontline Systems, Inc., que possibilita resolver problemas de até 8.000 variáveis e 8.000 restrições.

Um computador Dell Latitude D600 com processador Intel ${ }^{\circledR}$ Pentium $^{\circledR}, 1.6 \mathrm{GHz}$ e RAM de $512 \mathrm{Mb}$ foi usado com o sistema operacional Windows $\mathrm{XP}^{\circledR}$ da Microsoft. 0 tempo de processamento situou-se entre 30 e 40 segundos.

$\mathrm{O}$ DOH utilizado para as fábricas de produto acabado foi em torno de 70 dias. A alta cobertura de estoque nas fábricas se explica pelo perfil bastante sazonal da demanda e pelos grandes volumes de inventários em trânsito (importações). Para os centros de distribuição não se calculou o giro de estoque e, portanto, o inventário médio e, consequentemente, os custos dos inventários nesse elo da cadeia acabaram sendo considerados apenas parcialmente, já que apenas os parâmetros de custos de manuseio do estoque (handling) foram inseridos no modelo. Para essa aplicação, adotou-se um handling cost de 3,5\% para as fábricas e custos de oportunidade financeira de $11,5 \%$, conforme dados fornecidos pela empresa.

0 modelo mostrou-se bastante amigável e rápido para a solução do problema. Os resultados com a utilização da ferramenta foram significativos. A partir dos resultados da ferramenta em MSExcel após a otimização, pôde-se notar que o custo de material é o mais representativo, mas que, dentre os custos ligados à logística da empresa, a representatividade dos custos de carregamento de inventário é significativa. Esses custos de carregamento não eram considerados pelo pessoal de planejamento da empresa para definição da rede logística, já que a taxa de carregamento $\mathrm{CC}$ não era sequer calculada pela área financeira. Seguem as principais conclusões a partir das análises dos resultados:

- Dada a mesma demanda e distribuição de custos fixos e variáveis contempladas pelo modelo, comparou-se o custo total da empresa antes de se utilizar a ferramenta (definição empírica do modelo de suprimento) e o custo total a partir do uso da ferramenta computacional criada em MSExcel. A diferença sobre o custo total entre a rede logística original e a sugerida pela ferramenta se situou acima dos 5\%;

- Após a otimização, e mesmo sem considerar o custo de oportunidade financeira do inventário nos CDs (apenas os custos de manuseio dos estoques), o custo de inventário na cadeia situou-se em torno de $2 \%$ do total. Considerando apenas os custos fixos dos centros de distribuição, custos dos fretes de transferência e distribuição e os custos de carregamento de inventário (manuseio, seguro, oportunidade financeira), este último ficou em torno de $23 \%$ do custo total e, considerando apenas os custos variáveis (fretes, manuseio e oportunidade financeira), a representatividade dos custos de carregamento de inventário foi de 24,5\% do custo variável total;

- Os custos de carregamento de inventário foram significativamente maiores que os custos de frete de transferência entre as fábricas de produto intermediário e as fábricas de produto acabado; e

- Os custos de carregamento do inventário foram ainda maiores do que os custos fixos dos centros de distribuição. A percepção dessa representatividade dos custos do inventário não era tão clara antes da aplicação do modelo. 


\section{Conclusões}

A partir de uma análise na literatura disponível, pôde-se perceber uma lacuna nos modelos que se propõem a avaliar os custos de inventário na função objetivo para otimização de redes logísticas. Invariavelmente ou os custos de inventário são desconsiderados ou avaliados de forma parcial. Adotando-se os modelos de otimização encontrados na literatura, os custos de inventário seriam menores do que usando a metodologia aqui apresentada.

0 modelo demonstrou que se pode ter mais de 5\% de redução de custos comparando-se o resultado desse modelo com a rede logística inicialmente definida pela empresa. Mostrou-se também a importância atual dos estoques nos ativos dessa empresa e a sua representatividade quando se busca a otimização de uma rede logística, principalmente quando se tratar de produtos de alto valor agregado. $\mathrm{Na}$ empresa analisada, os custos de estoque se situaram em torno de um terço dos custos de fretes de transferência e distribuição, mesmo sem considerar o custo de oportunidade financeira nos centros de distribuição.

0 presente artigo apresenta uma metodologia simples incorporada a um modelo de localização de instalações com vários elos que permite a consideração do custo total de inventário a partir da cobertura de estoque em cada elo, mantendo a linearidade da função objetivo e das restrições. A metodologia permite o cálculo do inventário médio e de pico, com uma melhor avaliação do impacto dos custos totais de inventário e com a inclusão de restrições referentes à capacidade de armazenagem em fábricas e CDs.

Sugestões para desdobramento deste trabalho incluem:

- Uso da metodologia em outros problemas de definição de malha logística para certificação da aplicabilidade do modelo;

- Elaboração de tabela padrão por tipo de negócio/ instalação, para ser usada como referência para aplicação em modelos matemáticos que se utilizem da metodologia aqui sugerida;

- Estudo do impacto resultante da simplificação/ aproximação adotada - equação (7) - para empresas cujos custos dos produtos intermediários sejam muito diferentes por fábrica ou os custos de transportes entre $i$ e $j$ sejam significativos e muito diferentes; e

- Estudo comparativo entre os resultados dos custos de inventário, adotando-se a metodologia aqui apresentada e os custos resultantes de outras metodologias.

\section{Referências}

ARNTZEN, B. C. et al. Global Supply chain management at Digital Equipment Corporation. Interfaces, v. 25, n. 1, p. 69-93, 1995.

ARROW, K. A.; KARLIN, S.; SCARF, H. E. Studies in the Mathematical Theory of Inventory and Production. Stanford, CA, USA: Stanford University Press, 1958.

BALlOU, R. H. Business Logistics Management. 4 ed. Englewood Cliffs, NJ: Prentice-Hall, 1998.

BHUTTA, K. S. et al. An Integration Location, Production, Distribution and Investment Model for a Multinational Corporation. International Journal of Production Economics, v. 86, p. 201-216, 2003.

BHUTTA, K. S. Global Supply Chains: An Integration Location, Production, Distribution and Investment Model for a Multinational Corporation Operating Under Varying Exchange Rates and Tariff Structures. Dissertation (Doctoral)-University of Texas, Arlington, 2001.

BHUTTA, K. S. International facility location decisions: a review of the modelling literature. International Journal of Integrated Supply Management, v. 1, n. 1, p. 33-50, 2004.

BRITO JUNIOR, 1. Análise do impacto logístico de diferentes regimes aduaneiros no abastecimento de itens aeronáuticos empregando modelo de transbordo multiproduto com custos fixos. Dissertação (Mestrado)Escola Politécnica, Universidade de São Paulo, São Paulo, 2004.

CHRISTOPHER, M. Logística e Gerenciamento da Cadeia de Suprimentos - Estratégias para a redução de custos e melhoria dos serviços. São Paulo: Ed. Pioneira, 2002.

CLARK, A. J. A dynamic, single-item/multi-echelon inventory model. Santa Monica, California: The Rand Corporation, 1958. RM2297.

CRANDALL, R. E.; CRANDALL, W. R. Managing Excess Inventories: a life-cycle approach. Academy of Management Executive, v. 17, n. 3, 2003.

DOGAN, K.; GOETSCHALCKX, M. A primal decomposition method for the integrated design of multi-period production-distribution system. Research report. IIE Transactions, v. 31, n. 11, p. 1027-1036, 1999.

EFROYMSON, M. A.; RAY, T. L. A branch and bound algorithm for plant location. Operations Research, v. 14, p. 361-368, 1966.

EPPEN, G. D. Effects of centralization on the expected cost in a multi-location Newsboy Problem. Management Science, v. 25, p. 498-501, 1979.

FINANCIAL EDUCATION. Inventory Turnover and Days Inventory-On-Hand. 2007. Disponivel em: <http:// financial-education.com/2007/01/31/inventoryturnover-and-days-inventory-on-hand/>. Acesso em: dez. 2009.

FLIP0, C. D. Spatial decomposition for a multi-facility production and distribution problem. International Journal of Production Economics, v. 64, p. 177-186, 2000.

FLIPO, C. D.; FINKE, G. An integrated model for an industrial production-distribution problem. IIE Transactions, v. 33, n. 9, p. 705-716, 2001

GARCIA, E. S. et al. Gestão de estoques: otimizando a logística e a cadeia de suprimentos. Rio de Janeiro: E-papers Servicos Editoriais Ltda., 2006. Disponível em: <http:// 
books.google.com/books?id=AvfRM51NLcQC\&dq=ptPT>. Acesso em: dez. 2009.

GEOFFRION, A. M.; POWERS, R. F. Twenty years of strategic distribution system design: an evolutionary perspective. Interfaces, v. 25, n. 5, p. 105-127, 1995.

GOETSCHALCKX, M.; VIDAL, C. J.; DOGAN, K. Modeling and design of global logistics systems: A review of integrated strategic and tactical models and design algorithms. European Journal of Operational Research, v. 143, p. 1-18, 2002.

GROSS, D.; PINKUS, C. E.; SOLAND, R. M. Designing a multi-product/multi-echelon inventory system. In L.B. Schwartz, "Multi-commodity facilities location problem". Journal of the Operational Research Society, v. 32, p. 803-814, 1981.

HADJINICOLA, G. C.; KUMAR, K. R. Modeling manufacturing and marketing options in international operations. International Journal of Production Economics, v. 75, p. 287-304, 2002.

HAMAD, R.; GUALDA, N. D. F. Modelo para localização de instalações em escala global envolvendo quatro elos da cadeia logística. Transporte e Transformação, v. 10, p. 59-73, 2006

HAMAD, R.; GUALDA, N. D. F. Models for Facilities or Vendors Location in a Global Scale Considering Several Echelons in the Chain. Networks and Spatial Economics, v. 8, p. 297-307, 2008.

HARRIS, F. W. How many parts to make at once. Factory, The Magazine of Management, v. 10, n. 2, p. 135-136, 1913.

KUEHN, A. A.; HAMBURGER, M. J. A heuristic program for locating warehouses. Management Science, v. 9, p. 643-666, 1963.

MOHAMED, Z. An integrated production-distribution model for a multinational company operating under varying exchange rates. International Journal of Production Economics, v. 58, p. 81-92, 1999.

NAHMIAS, S. Single-Product, Single-Location Models. In: GRAVES, S. C. et al. Handbooks in OR \& MS. 1993. chap. 1, p. 3-55.

NOZICK, L. K.; TURNQUIST, M. A. Inventory Transportation, Service Quality and the Location of Distribution Centers. European Journal of Operations Research, v. 129, p. 362-371, 2001.

OWEN, S. H.; DASKIN, M. S. Strategic facility location: A review. European Journal of Operational Research, v. 111, p. 423-447, 1998.

REVELLE, C. S.; EISELT, H. A. Location analysis: A synthesis and survey - Invited review. European Journal of Operational Research, v. 165, p. 1-19, 2005.

SHAPIRO, J. F. Modeling the Supply Chain. Pacific Grove CA Duxbury Press, 2001.

SMITS, S. R. Evaluation model for the design of Distribution Networks. The Netherlands: Faculty of Technology Management, 2001.

SPIELBERG, K. Algorithms for the simple plant-location problem with some side constraints. Operations Research, v. 17, p. 85-111, 1969.

STANDAGE, T. An edible history of humanity. Walker \& Company, 2009. 288 p.

SYAM, S. S. A model and methodologies for the location problem with logistical components. Computers and Operations Research, v. 29, p. 1173-1193, 2002.

VALLIM FILHO, A. R. A. Localização de Centros de Transporte e Carga - Uma contribuição para Modelos de Otimização. Tese (Doutorado)-Escola Politécnica, Universidade de São Paulo, São Paulo, 2004.

WILSON, R. H. A Scientific Routine for Stock Control. Harvard Business Review, v. 13, p. 116-128, 1934.

\title{
Logistic networks modeling with inventory carrying costs calculated using inventory turnover
}

\begin{abstract}
Inventory carrying cost has become very important in the analysis of the trade-offs and an important component to be considered when taking decisions about a logistic network. This paper proposes a methodology incorporated in a multi-echelon sourcing decision model, to be considered as a new version of the model presented in Hamad, Gualda (2008). It treats the carrying costs using the inventory Days-on-hand estimate in each echelon of the chain (plants and/or Distribution Centers). The main contributions of this methodology compared to other options found in the literature are the simplicity of its application, the consideration of all inventory costs (not only the ones related to the products being modeled) and, also, the inclusion of constraints related to warehousing capacity.
\end{abstract}

\section{Keywords}

Logistic networks optimization. Logistics network design. Inventory turnover. Inventory carrying costs. 